\title{
No positive effect of rhdnase on the pulmonary colonization in children with cystic fibrosis
}

\author{
H.J.C. Bonestroo, M.G. Slieker, H.G.M. Arets
}

ABSTRACT: No positive effect of rhdnase on the pulmonary colonization in children with cystic fibrosis. H.J.C. Bonestroo, M.G. Slieker, H.G.M. Arets.

Background. Long-term clinical trials have shown that daily treatment with recombinant human deoxyribonuclease (rhDNAse) in patients with mild to moderate cystic fibrosis (CF) improves lung function and decreases the number of respiratory exacerbations. The aim of this study was to analyze the effect of rhDNAse on the bacterial colonization of the airways in children with $\mathrm{CF}$.

Methods. This was a retrospective cohort study. From the database of the CF Center Utrecht, we selected two groups, an rhDNAse group (daily $2.5 \mathrm{mg}$ rhDNAse) and a control group (no rhDNAse). Primary outcome parameter was the difference in change in bacterial colonization between the treatment and control group during 1.5-year. Secondary outcome parameters were changes in lung function $\left(\mathrm{FEV}_{1}\right)$ and pulmonary exacerbations.

Results. Children treated with rhDNAse showed no significant changes in bacterial colonization during the treatment period, apart from an increase of $P$. aeruginosa positive cultures, both compared to baseline $(53.1 \%$ versus $25 \%, p<0.05$ ) and control group (no change during study period, $37 \%$ versus $37 \%$ ). The change in $\mathrm{FEV}_{1}$ after one year of treatment was $+4.0 \%$ in the treatment group versus $-0.3 \%$ in the control group $(p=0.22)$. There were no significant changes in number of pulmonary exacerbations.

Conclusions. This study showed no significant beneficial decrease in bacterial airway colonization during 1.5-year of treatment with rhDNAse. The positive effects of rhDNAse on the lung function can therefore not be explained by a change in airway colonization.

Monaldi Arch Chest Dis 2010; 73: 1, 12-17.

Keywords: Cystic Fibrosis, rhDNAse, Pseudomonas aeruginosa, Pulmonary colonization.

Department of Pediatric Pulmonology, University Medical Center Utrecht, the Netherlands.

Correspondence: H.G.M. Arets, MD, PhD., Department of Pediatric Pulmonology, University Medical Center Utrecht, Room KH 01.419.0, P.O. Box 85090, 3508 AB Utrecht, The Netherlands; e-mail: h.arets@umcutrecht.nl

\section{Introduction}

Cystic fibrosis (CF) is a multi-system disorder, but progressive respiratory disease is the most important cause of morbidity and mortality [1]. It is characterized by progressive impairment in lung function and airway obstruction with recurrent airway infection, caused by accumulation of thick viscous purulent secrete [2]. The most important microorganisms causing airway infections in young children with CF are Staphylococcus aureus (SA) and Haemophilus influenzae (HI) [3-5]. Pseudomonas aeruginosa (PA) will be cultured from the majority of patients during later life [6]. Mucociliary clearance of the viscous mucus is impaired, which causes an ideal basis for colonization and infection with microorganisms. These infections give local inflammation with an influx of predominantly neutrophils $[7,8]$. Degradation of these neutrophils causes DNA release, which leads to an increase in viscosity of sputum, further increasing the susceptibility for colonization and infection [9]. In this way the vicious circle of infection, inflammation and obstruction leading to a progressive loss of lung function and airway destruction is maintained.
The treatment of $\mathrm{CF}$ is especially focused on the prevention of lung disease and prevention of lung function loss. Important components of this treatment are antimicrobial agents, mucolytics and physiotherapy. Since 1992 an increasing number of patients has been treated with nebulized recombinant human deoxyribonuclease (rhDNAse) [10]. RhDNAse breaks down extra cellular DNA, thereby decreasing the viscosity of sputum. Several long term clinical trials showed that rhDNAse treatment is effective, especially in mild to moderate $\mathrm{CF}$, shown by an increase in lung function and a decrease of respiratory exacerbations [10-19]. A study preformed in Denmark hypothesized that this clinical improvement in lung function might be due to a decrease in bacterial airway colonization [11]. In this study a decrease in bacterial colonization was reported, but only CF patients without bronchopulmonary infection or chronic bronchorrhoea where included. However, in most hospitals rhDNAse is only started in children with chronic bronchorrhoea and/or chronic bronchopulmonary infection. Besides, in Denmark, the antibiotic treatment regimes are stricter than and different from the regimes in many other countries [20]. Therefore, results from the Danish study might not 
be representative for the situation in other countries, such as the Netherlands. We investigated the effect of rhDNAse treatment on the bacterial airway colonization in a group of children with $\mathrm{CF}$, in whom treatment was started on clinical grounds. Secondly, we investigated if treatment with rhDNAse leads to a change in lung function and respiratory exacerbations.

\section{Methods}

\section{Subjects and Design}

Data were obtained from CF patients during routine yearly controls. For this retrospective chart analysis ethical approval was waived by our IRB. In total 260 children from the central Netherlands are treated in the CF center Utrecht. All patients undergo a routine yearly multidisciplinary examination regardless of their health status. Since 1997 , all results have routinely been collected in an electronic database. From this database, we selected all children with CF (diagnosis based on clinical symptoms and positive sweat testing and/or two CF causing DNA mutations) under the age of 20 years, starting rhDNAse treatment between January 2004 and December 2006 and continuing this treatment for at least 1.5-year. The decision to commence rhDNAse was at the discretion of the attending pediatricians, based on clinically diagnosed chronic bronchorrhoea, most often combined with signs of chronic bronchial infection. In most children, the effect of rhDNAse was evaluated after 3 to 6 months based on pulmonary function or subjective changes in symptoms. The clinical parameters were collected from 1 year before start of rhDNAse treatment until 1.5-year after starting the treatment. For every patient using rhDNAse, we selected a control patient from the database, matched for the nearest age and sex. From these controls, data were also available for the above-mentioned period. Selection was independent of disease severity, the presence of chronic bronchopulmonary colonization or the use of antibiotics.

\section{Microbiology}

Cultures were taken from sputum. If the patient was unable to produce sputum a cough swab was collected. Only if patients were not able to cough on demand, throat swabs were taken as best alternative. From all patients the last three cultures were taken the year before starting rhDNAse treatment and all cultures during 1.5-year after start of rhDNAse. Cultures were considered positive when minimal one sample presented one or more of the following microorganisms: PA, SA, HI, $S$. pneumoniae, $C$. albicans, A. fumigatus or $S$. maltophilia. In the control patients, cultures were analyzed over the same period.

\section{Antimicrobial treatment}

Pulmonary exacerbations were defined as any deterioration of symptoms and pulmonary function requiring antibiotic treatment as judged by the attending pediatrician. Antibiotic treatment was based on available sputum culture results. At the beginning of treatment new sputum sample were collected. The number of antibiotic courses was used as a proxy of the number of pulmonary exacerbations. Antimicrobial treatment were administrated for at least 2 weeks, principally through oral medication. Severe exacerbations or those not responding to oral courses of antibiotics were treated with intravenous antibiotics over a period of 2-3 weeks. The treatments were based on susceptibility to antibiotics. Patients with frequent and/or chronic upper or lower airway infections were prophylactically treated with cotrimoxazol or cefuroxim in patients without PA, and with azitromycine +/- tobramycin or colistin nebulisation in those patients colonized with PA.

\section{Lung function tests}

Lung function was measured in all children from 4 years of age onwards using spirometry in conformity with international criteria [21]. For the measurements, a Lilly pneumotachometer system (Viasys, Masterscreen, Hochberg, Germany), was used and sex and height specific reference values were applied [22]. The primary lung function parameter was forced expiratory volume in one second as percentage of predicted $\left(\mathrm{FEV}_{1} \%\right.$ pred). From the $\mathrm{CF}$ database from every patient one $\mathrm{FEV}_{1}$ before starting rhDNAse and one 1-1.5 year after start of rhDNAse were used. The same parameters were analyzed for the control patients at corresponding age.

\section{Data Analysis}

Data were analyzed with SPSS for windows. Differences between patients with and without rhDNAse treatment were calculated using McNemar test for discrete variables, and paired Student's $t$-test for normally distributed continuous variables. Chisquare was used to compare dichotomous variables. Mann-Whitney U test was used to compare not normally distributed continuous data. To adjust for potential confounders, multivariate analyzes were performed using multiple linear regression. A $p$-value of $<0.05$ was accepted as statistically significant.

\section{Results}

\section{Patients}

Between January 2004 to December 2006, seventy-three children used rhDNAse. Thirty-five infants were included in the study. Of the excluded infants, ten patients used rhDNAse less than 1.5year, nineteen patients did not use rhDNAse on a regular basis, in four patients sufficient data were not available and five patients were older than 20 years. For the thirty-five patients using rhDNAse, thirty-five gender and age matched control patients were selected. 
Table 1 shows the baseline characteristics of both groups before the start of rhDNAse treatment. There were no significant differences in characteristics between the groups, except for lung function. Before the start of rhDNAse, the treatment group had a significantly lower $\mathrm{FEV}_{1}$ $\%$ pred compared to the control group $(78.1 \%$ versus $97.7 \%, p<0.05)$. In the control group, eight patients were prophylactically treated with cotrimoxazol or cefuroxim and 17 with azitromycine +/- tobramycin or colistin nebulization compared with 11 and 21 patients in the treatment group, respectively. This difference was not significant.

\section{Culture results}

The percentage of positive cultures for all bacteria before starting rhDNAse was significantly higher in the treatment group compared to the control group, $94 \%$ versus $84 \%, p<0.05$. During study period, no significant changes in colonization were seen in both the treatment (94\% versus 96\%) and control group (84\% versus $88 \%$ ). Before treatment the percentage of positive cultures for individual bacteria was not significant different between the treatment and control group. The prevalence of PA positive cultures increased significantly during the study period in the treatment group (25\% to $53.1 \%$, $p<0.05$ ) (table 2). During study period, no significant changes were seen in the control group (37\% versus $37 \%, p=1.00)$. For all other microorganisms, no significant changes were found either within or between the two groups (table 2).

\section{Lung function and respiratory exacerbations}

Both before and after treatment $\mathrm{FEV}_{1} \%$ pred was significant lower in the treatment group compared to the controls. The absolute change of $\mathrm{FEV}_{1} \%$ pred after a year of rhDNAse treatment was $+4.0 \%$ in the treatment group versus $-0.3 \%$ in the control group ( $p=0.22)$ (figure 1). Multivariate analysis showed that the independent effect of rhDNAse on lung function was greater among infants colonized with PA compared to noncolonized infants, but this difference was not statistic significant $(13.3 \%$ vs $4.8 \%, p=0.40)$. Compared to the control patients, the patients in the treatment group showed more pulmonary exacerbations that prompted antibiotic courses in the year prior to rhDNAse treatment (2.40 versus 1.26 courses, $p<0.05$ ). The exacerbation rate in both treatment and control group did not change during 1.5 -year of treatment, however the difference in exacerbation rate during treatment was not significant anymore (2.46 versus $1.51, \mathrm{p}=0.11$ )

\section{Discussion}

In this study, we found that CF children treated with rhDNAse showed no significant changes in bacterial colonization during the treatment period, apart from an (unexpected) increase in PA colonization. Besides, children with $\mathrm{CF}$ tended to have better lung functions after rhDNAse treatment.

RhDNAse treatment in $\mathrm{CF}$ has been shown to be effective, improving lung function and decreasing the number of respiratory exacerbations [11-19]. The exact reason for this positive effect remains unclear. $\mathrm{CF}$ is characterized by an abnormal rheology of the mucus layer [23, 24], due to the presence of an increased amount of DNA, originating especially from neutrophils $[7,8]$. The effect of rhDNAse is based on depolymerization of this DNA [25]. In most studies a decrease of viscoelasticity, improving mucus transport and enabling sputum expectoration with or without physiotherapeutic maneuvers is facilitated [26]. This mechanism is supposed to reduce the induced chance of infection exacerbations $[12,13,18]$. Direct anti-inflammatory effects of rhDNAse have also been described, for instance reflected by a decrease in IL-8 [27].

Table 1. - Baseline characteristics of patients treated with rhDNAse compared to control patients not receiving rhDNAse

\begin{tabular}{lccc}
\hline Characteristics & $\begin{array}{c}\text { rhDNAse } \\
(\mathrm{n}=35)\end{array}$ & $\begin{array}{c}\text { Controls } \\
(\mathrm{n}=35)\end{array}$ & $p$-value \\
\hline Female gender & $17(49 \%)$ & $17(49 \%)$ & 1.00 \\
\hline $\begin{array}{l}\text { Age }(\text { years }) \\
\text { range }\end{array}$ & $\begin{array}{c}\text { (.4 } \\
1.7-17.7\end{array}$ & $\begin{array}{l}9.5 \pm 4.0 \\
2.8-18.1\end{array}$ & 0.93 \\
\hline $\begin{array}{l}\text { Height }(\mathrm{cm}) \\
\text { Weight }(\mathrm{Kg})\end{array}$ & $134.0 \pm 24.4$ & $133.6 \pm 21.7$ & 0.95 \\
\hline BMI $\left(\mathrm{Kg} / \mathrm{m}^{2}\right)$ & $30.8 \pm 12.7$ & $31.7 \pm 14.6$ & 0.79 \\
\hline FEV $(\%$ pred $)$ & $16.4 \pm 1.7$ & $16.8 \pm 2.5$ & 0.43 \\
\hline range & $78.1 \pm 16.9$ & $97.7 \pm 18.1$ & $<0.05$ \\
\hline
\end{tabular}

Data are given as numbers and percentages or mean \pm standard deviation.

BMI: body mass index; $\mathrm{FEV}_{1}$ : forced expiratory volume in one second expressed as $\%$ of predicted values; $\mathrm{N}$ : number of patients; rhDNAse: recombinant human deoxyribonuclease. 
Table 2. - Number of patients with positive culture results in the rhDNAse group and control group before start of rhDNAse treatment compared to culture results after 1.5-yr study period

\begin{tabular}{lcccccc}
\hline \multirow{2}{*}{ Microorganism } & \multicolumn{2}{c}{ rhDNAse group $(\mathrm{n}=32)$} & \multicolumn{2}{c}{ Control group $(\mathrm{n}=27)$} \\
\cline { 2 - 7 } & Before & After & $p$-value & Before & After & $p$-value \\
\hline P. aeruginosa & $8(25 \%)$ & $17(53 \%)$ & $<0.05$ & $10(37 \%)$ & $10(37 \%)$ & n.s. \\
\hline S. aureus & $23(72 \%)$ & $22(69 \%)$ & n.s. & $18(67 \%)$ & $17(63 \%)$ & n.s. \\
\hline S. pneumoniae & $0(0 \%)$ & $0(0 \%)$ & - & $2(7 \%)$ & $1(4 \%)$ & n.s. \\
\hline H. influenzae & $8(25 \%)$ & $7(22 \%)$ & n.s. & $3(11 \%)$ & $5(19 \%)$ & n.s. \\
\hline C. albicans & $17(53 \%)$ & $23(72 \%)$ & n.s. & $12(44 \%)$ & $16(59 \%)$ & n.s. \\
\hline A. fumigatus & $12(38 \%)$ & $16(50 \%)$ & n.s. & $7(26 \%)$ & $8(29 \%)$ & n.s. \\
\hline S. maltophilia & $4(13 \%)$ & $5(16 \%)$ & n.s. & $1(4 \%)$ & $1(4 \%)$ & n.s. \\
\hline
\end{tabular}

Data are given as numbers and percentages of patients with positive culture results.

After: number of patients with positive culture results after 1.5-yr study period; Before: number of patients with positive culture results before treatment; n.s.: not significant; rhDNAse: recombinant human deoxyribonuclease.

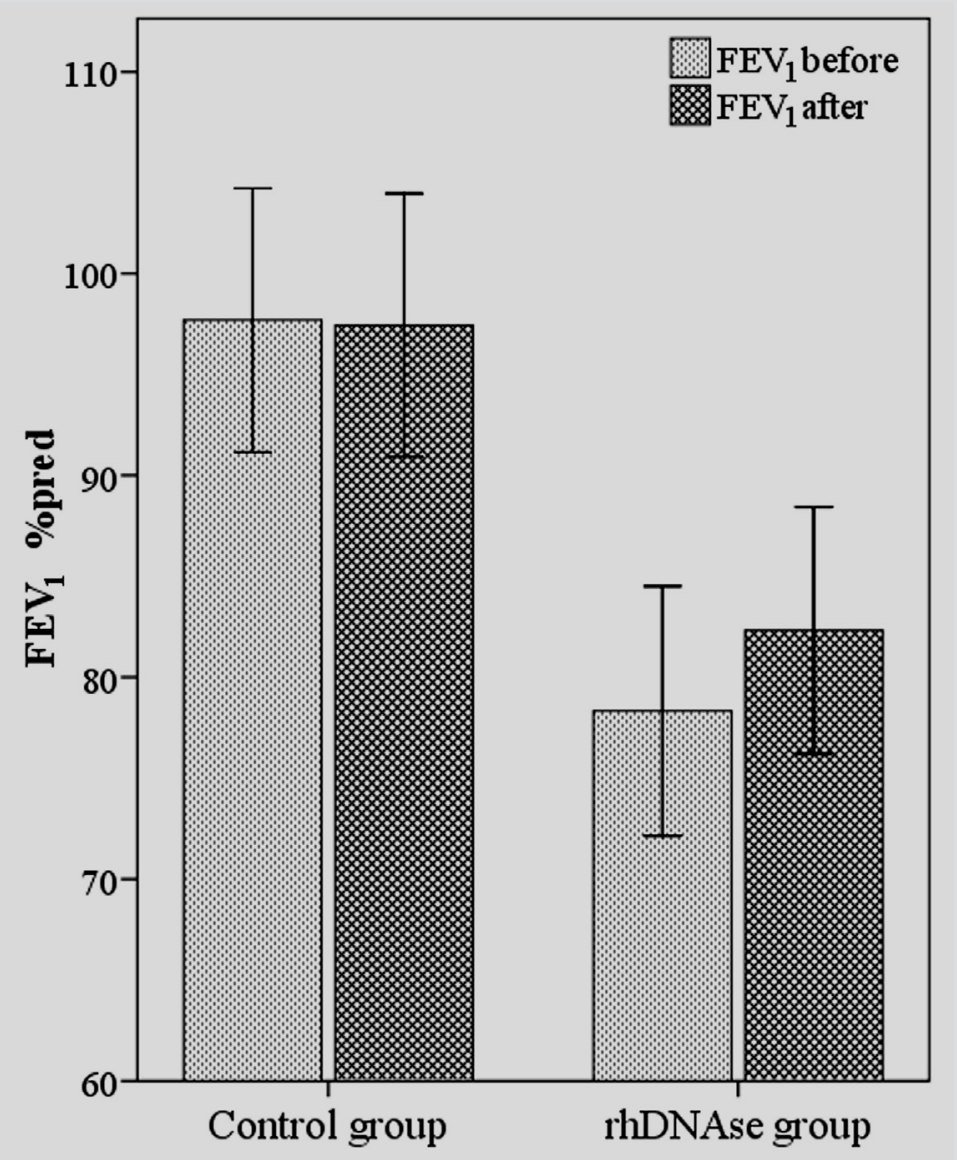

Fig. 1. - Mean lung function of the control and rhDNAse group before rhDNAse treatment and after 1.5 -year study period. I: $95 \%$ confidence interval. $\mathrm{FEV}_{1}$ : forced expiratory volume in one second expressed as $\%$ of predicted values for sex/age specific reference value. FEV $_{1}$ after: lung function after 1.5-year study period. $\mathrm{FEV}_{1}$ before: lung function before treatment.
Most exacerbations in $\mathrm{CF}$ patients are caused by bacterial infections, especially SA and PA [3-6]. A study from Denmark proposed that the decrease of exacerbation rate in patients using rhDNAse might be caused by a decrease in the bacterial airway colonization [11]. The investigators found that in children with $\mathrm{CF}$, with mild to moderate pulmonary function abnormalities, the number of positive cultures in the untreated group was significantly higher than in the treated group. This effect was especially seen for SA. The investigators found no significant difference for PA [11]. We could not reproduce their findings in the present study; we found no decrease in the numbers of positive cultures. Moreover, there even was a significant increase in the prevalence of PA after 1.5-year treatment with rhDNAse.

The differences between the study results can be explained by the difference in patient populations. The Danish study included CF patients without chronic bacterial pulmonary infection for rhDNAse treatment, a group of patients who are not treated with rhDNAse in many other countries, such as the Netherlands. In our study rhDNAse treatment was limited 
to patients with clinical indications for treatment, namely children with chronic bronchorrhoea and/or chronic bronchopulmonary infection. The second explanation for the difference in outcome might be that Danish antibiotic regimes are different from those in the Netherlands [20]. Patients in the Danish study were not on maintenance antibiotic treatment. In the Netherlands, most patients with chronic bronchorrhoea and chronic bronchopulmonary infections are on maintenance treatment. Third, the prevalence and resistance of microorganisms in Denmark differs from those in the Netherlands [11, 28, 29]. Last, the Danish study was a prospective randomized case-control study, the present study a retrospective cohort study. This retrospective design and the relatively limited number of patients does of course impair any randomization, but on the other hand has the advantage to reflect daily practice.

Some reasons for the surprising increase in prevalence of PA after rhDNAse can be considered. First, as might be expected, the treatment group had a significant lower lung function before starting rhDNAse, and more respiratory exacerbations during the year prior to treatment. Therefore, the treatment group probably was more vulnerable and had a priori increased risk for PA contamination and colonization than the control group. Secondly, by using rhDNAse better and increased sputum expectoration was established, increasing the chance to have PA positive cultures compared to spontaneous sputum expectoration. A third explanation for the rise of PA in the treatment group might be that the nebulization process increases the risk of PA contamination [30]. During nebulization there is a direct passage to the airways, which may increase the risk of inhalation of microorganisms such as PA.

Secondly, we investigated the effect of rhDNAse on lung function and respiratory exacerbations. After 1.5-year of rhDNAse treatment, we found a $4 \%$ increase in $\mathrm{FEV}_{1}$ in the treatment group. In the control group a $0.3 \%$ decrease was found after 1.5 -year study period. Therefore, changes in lung function were much better in the treatment group compared to the control group. However, this difference was not significant within or between the groups. An increase in antibiotic courses in both groups was seen during study period, however the increase was higher in the control group compared to the treatment group, but this also lacked significance. Several trails with large study populations showed a significant effect on the number of antibiotic courses and lung function increase $[11-16,18,19,26]$. We could not find these significant differences, probably because of the relatively small number of participants in the present study.

In conclusion, in this retrospective "every-daylife" study, representing the clinical practice of Dutch CF care, we found that $\mathrm{CF}$ patients treated with rhDNAse for 1.5-year showed no significant decrease in bacterial colonization, and even an increase in $P$. aeruginosa colonization. Therefore we cautiously conclude that the positive effects of
rhDNAse on lung function can not be explained by a change in bacterial airway colonization.

\section{References}

1. Menzin J, Oster G, Davies L, et al. A multinational economic evaluation of rhDNase in the treatment of cystic fibrosis. Int J Technol Assess Health Care 1996; 12: 52-61.

2. Gibson RL, Burns JL, Ramsey BW. Pathophysiology and management of pulmonary infections in cystic fibrosis. Am J Respir Crit Care Med 2003; 168: 918-951.

3. Khan TZ, Wagener JS, Bost T, Martinez J, Accurso FJ, Riches DW. Early pulmonary inflammation in infants with cystic fibrosis. Am J Respir Crit Care Med 1995; 151: 1075-1082.

4. Rosenfeld M, Gibson RL, McNamara S, et al. Early pulmonary infection, inflammation, and clinical outcomes in infants with cystic fibrosis. Pediatr Pulmonol 2001; 32: 356-366.

5. Armstrong DS, Grimwood K, Carlin JB, Carzino R, Olinsky A, Phelan PD. Bronchoalveolar lavage or oropharyngeal cultures to identify lower respiratory pathogens in infants with cystic fibrosis. Pediatr Pulmonol 1996; 21: 267-275.

6. Robertson JM, Friedman EM, Rubin BK. Nasal and sinus disease in cystic fibrosis. Paediatr Respir Rev 2008; 9: 213-219.

7. Chernick WS, Barbero GJ. Composition of tracheobronchial secretions in cystic fibrosis of the pancreas and bronchiectasis. Pediatrics 1959; 24: 739-745.

8. Smith AL, Redding G, Doershuk C, et al. Sputum changes associated with therapy for endobronchial exacerbation in cystic fibrosis. J Pediatr 1988; 112: 547-554

9. Chmiel JF, Davis PB. State of the art: why do the lungs of patients with cystic fibrosis become infected and why can't they clear the infection? Respir Res 2003; 4: 8.

10. Jones AP, Wallis CE. Recombinant human deoxyribonuclease for cystic fibrosis. Cochrane Database Syst Rev 2003; CD001127.

11. Frederiksen B, Pressler T, Hansen A, Koch C, Hoiby N. Effect of aerosolized rhDNase (Pulmozyme) on pulmonary colonization in patients with cystic fibrosis. Acta Paediatr 2006; 95: 1070-1074.

12. Fuchs HJ, Borowitz DS, Christiansen DH, et al. Effect of aerosolized recombinant human DNase on exacerbations of respiratory symptoms and on pulmonary function in patients with cystic fibrosis. The Pulmozyme Study Group. N Engl J Med 1994; 331: 637-642.

13. Quan JM, Tiddens HA, Sy JP, et al. A two-year randomized, placebo-controlled trial of dornase alfa in young patients with cystic fibrosis with mild lung function abnormalities. J Pediatr 2001; 139: 813-820.

14. Laube BL, Auci RM, Shields DE, et al. Effect of rhDNase on airflow obstruction and mucociliary clearance in cystic fibrosis. Am J Respir Crit Care Med 1996; 153: 752-760.

15. Ramsey BW, Astley SJ, Aitken ML, et al. Efficacy and safety of short-term administration of aerosolized recombinant human deoxyribonuclease in patients with cystic fibrosis. Am Rev Respir Dis 1993; 148: 145-151.

16. Ranasinha C, Assoufi B, Shak S, et al. Efficacy and safety of short-term administration of aerosolised recombinant human DNase I in adults with stable stage cystic fibrosis. Lancet 1993; 342: 199-202.

17. Shah PI, Bush A, Canny GJ, Colin AA, et al. Recombinant human DNase I in cystic fibrosis patients with severe pulmonary disease: a short-term, doubleblind study followed by six months open-label treatment. Eur Respir J 1995; 8: 954-958. 
18. McCoy K, Hamilton S, Johnson C. Effects of 12-week administration of dornase alfa in patients with advanced cystic fibrosis lung disease. Pulmozyme Study Group. Chest 1996; 110: 889-895.

19. Robinson PJ: Dornase alfa in early cystic fibrosis lung disease. Pediatr Pulmonol 2002; 34: 237-241.

20. Hansen CR, Pressler T, Hoiby N. Early aggressive eradication therapy for intermittent Pseudomonas aeruginosa airway colonization in cystic fibrosis patients: 15 years experience. J Cyst Fibros 2008; 7: 523-530.

21. Arets HG, Brackel HJ, van der Ent CK. Forced expiratory manoeuvres in children: do they meet ATS and ERS criteria for spirometry? Eur Respir J 2001; 18: 655660.

22. Zapletal A, Samanek M, Paul T: Lung function in children and adolescents. Methods, reference values. In: Zapletal A, ed. Progress in Respiration Research. Basel, Switzerland, Karger, 1987; 22: 114-218.

23. Rowe SM, Miller S, Sorscher EJ. Cystic fibrosis. $N$ Engl J Med 2005; 352: 1992-2001.

24. Ratjen F, Doring G. Cystic fibrosis. Lancet 2003; 361: 681-689.

25. Shak S, Capon DJ, Hellmiss R, Marsters SA, Baker CL. Recombinant human DNase I reduces the viscosity of cystic fibrosis sputum. Proc Natl Acad Sci USA 1990; 87: 9188-9192.

26. Shah PL, Scott SF, Knight RA, Marriott C, Ranasinha C, Hodson ME. In vivo effects of recombinant human DNase I on sputum in patients with cystic fibrosis. Thorax 1996; 51: 119-125.

27. Paul K, Rietschel E, Ballmann M, et al. Effect of treatment with dornase alpha on airway inflammation in patients with cystic fibrosis. Am J Respir Crit Care Med 2004; 169: 719-725.

28. Ciofu O, Fussing V, Bagge N, Koch C, Hoiby N. Characterization of paired mucoid/non-mucoid Pseudomonas aeruginosa isolates from Danish cystic fibrosis patients: antibiotic resistance, beta-lactamase activity and RiboPrinting. J Antimicrob Chemother 2001; 48: 391-396.

29. Johansen HK, Kovesi TA, Koch C, Corey M, Hoiby N, Levison H. Pseudomonas aeruginosa and Burkholderia cepacia infection in cystic fibrosis patients treated in Toronto and Copenhagen. Pediatr Pulmonol 1998; 26: 89-96.

30. Monforte V, Roman A, Gavalda J, et al. Contamination of the nebulization systems used in the prophylaxis with amphotericin B nebulized in lung transplantation. Transplant Proc 2005; 37: 4056-4058.

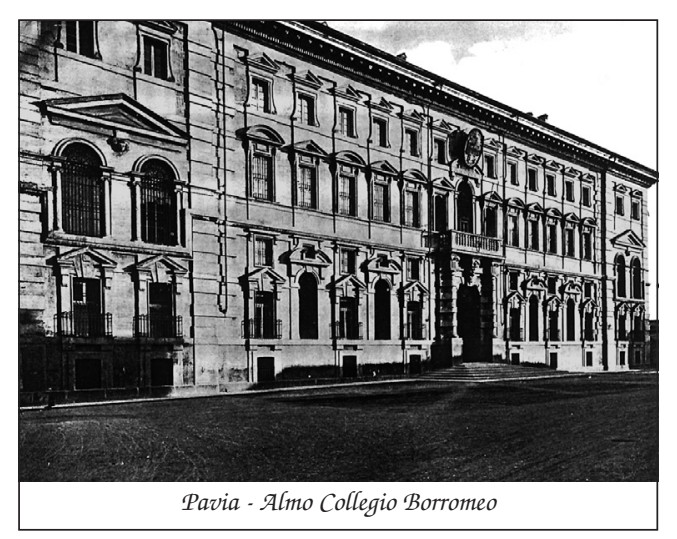

